\title{
Strong Correlation Effects on Topological Quantum Phase Transitions in Three Dimensions
}

\author{
A. Amaricci ${ }^{1}$, J. C. Budich ${ }^{2,3}$, M. Capone ${ }^{1}$, B. Trauzettel ${ }^{4}$, and G. Sangiovanni ${ }^{4}$ \\ ${ }^{1}$ Democritos National Simulation Center, Consiglio Nazionale delle Ricerche, \\ Istituto Officina dei Materiali (IOM) and Scuola Internazionale Superiore \\ di Studi Avanzati (SISSA), Via Bonomea 265, 34136 Trieste, Italy \\ ${ }^{2}$ Institute for Theoretical Physics, University of Innsbruck, 6020 Innsbruck, Austria \\ 3 Institute for Quantum Optics and Quantum Information, \\ Austrian Academy of Sciences, 6020 Innsbruck, Austria and \\ ${ }^{4}$ Institut für Theoretische Physik und Astrophysik, \\ Universität Würzburg, Am Hubland, D-97074 Würzburg, Germany
}

(Dated: June 9, 2016)

\begin{abstract}
We investigate the role of short-ranged electron-electron interactions in a paradigmatic model of three dimensional topological insulators, using dynamical mean-field theory and focusing on non magnetically ordered solutions. The non-interacting band-structure is controlled by a mass term $M$, whose value discriminates between three different insulating phases, a trivial band insulator and two distinct topologically non-trivial phases. We characterize the evolution of the transitions between the different phases as a function of the local Coulomb repulsion $U$ and find a remarkable dependence of the $U-M$ phase diagram on the value of the local Hund's exchange coupling $J$. However, regardless the value of $J$, following the evolution of the topological transition line between a trivial band insulator and a topological insulator, we find a critical value of $U$ separating a continuous transition from a first-order one. When the Hund's coupling is significant, a Mott insulator is stabilized at large $U$. In proximity of the Mott transition we observe the emergence of an anomalous "Mott-like" strong topological insulator state.
\end{abstract}

PACS numbers: 03.65.Vf, 71.10.Fd, 05.30.Rt, 71.30.+h

\section{INTRODUCTION}

Recently, the conventional Landau classification of matter has been complemented by the concept of topological phases. Rather than being distinguished by the value of local order parameters reflecting spontaneously broken symmetries, topological phases of matter are characterized by global invariants describing topological properties of the many-body wave-function. In the context of band structure physics, the systematic search for topological phases resulted in the discovery of a periodic table $^{1,2}$ that builds upon the Altland-Zirnbauer symmetry classification ${ }^{3}$.

In two spatial dimensions (2D), the seminal theoretical $^{4-6}$ and experimental ${ }^{7}$ discovery of time reversal symmetry (TRS) protected topological insulators (TIs) has inspired the theoretical community to analyze the influence of Coulomb repulsion on such systems. Evidently, the interplay between significant Coulomb interactions and topological aspects of matter holds the promise of nontrivial and intriguing effects emerging from the competition between the localization tendency induced by strong correlations and the peculiar band-structure of TIs. Regarding the influence of electron-electron interactions on 2D TIs, a wealth of results has been accumulated focusing on several model Hamiltonians with different techniques (for a recent review see e.g. Ref. 8). Among others, the effect of strong correlations on quantum spin Hall insulators and the related topological transitions have been addressed using quantum Monte-Carlo calculations ${ }^{9-11}$, various cluster based approaches $^{12-16}$, as well as dynamical mean field theory (DMFT) and extensions ${ }^{17-25}$. Interestingly, the possibility of inducing the formation of a topological phase by means of electronic repulsion has also been $\operatorname{proposed}^{26,27}$.

In three spatial dimensions (3D), symmetry-protected TIs have been have been suggested theoretically ${ }^{28-31}$ only about two years later than their counterparts in 2D. Remarkably, 3D TIs that preserve TRS exhibit nondegenerate Dirac fermion surface states. This feature makes them particularly appealing for fundamental science questions related to relativistic quantum mechanics. Soon after their theoretical prediction, these topological phases of matter have been experimentally detected in three-dimensional calchogenides ${ }^{32-36}$.

The interplay of strong electronic correlations with the various topological phases, realizable in $3 \mathrm{D}^{28}$, pledges to unlock access to a large variety of possible anomalous states. For instance, some Iridate compounds, with a pronounced three-dimensional character and the concomitant presence of strong spin-orbit coupling and sizeable electron-electron interaction, have been proposed either as correlated $3 \mathrm{D} \mathrm{TIs}^{37}$ or to host even more exotic, fractionalized topological states ${ }^{38,39}$. The role of interactions in enhancing the effect of spin-orbit coupling has been proposed as key to understand the onset of a topological Kondo insulating state in both $\mathrm{SmB}_{6}$ and $\mathrm{PuB}_{6}{ }^{40-44}$. Additionally, an interaction driven $3 \mathrm{D}$ topological Mott insulating state has been discussed in Refs. 45 and 46, while the existence of a U(1) spin-liquid 
with non-trivial spinon gap has been proposed in Ref.37 for a class of pyrochlore Iridates. More recently a Platinum oxide, $\mathrm{Ca}_{2} \mathrm{PtO}_{4}$, has also been suggested to become a $d$-electron weak TI if doped with holes ${ }^{47}$. Moreover, the realization of 3D TIs in oxides ${ }^{48}$, which have typically sizeable values of the interaction parameters, gives a further motivation to study the interplay of topology and electronic interactions. However, the more complicated topological structure of the 3D TIs with respect the their 2D counterparts has not yet allowed for a systematic study of correlated effects in 3D.

In this work, we present a thorough study of the effects of a local electronic interaction on the properties of a paradigmatic model of a 3D TI. Using DMFT, we solve non-perturbatively a two-band Hubbard model in presence of a multi-orbital density-density interaction, featuring a Hund's coupling term. We characterize the evolution of the non-trivial topological states of the system as a function of the interaction strength. We obtain the phase-diagrams of the model in the two-dimensional space spanned by the Hubbard repulsion and the crystalfield splitting, and determine the transition lines between insulating phases with different topological properties. One of the most prominent features of the model is that the transition line between the trivial band insulator and the strong topological insulator is divided in two parts by a critical point.

For weak interactions the phase-transition is adiabatically connected with the non-interacting transition and it is continuous. On the contrary, in the large interaction regime beyond the critical point, the topological quantum phase transition (TQPT) becomes of first-order. Unlike the conventional scenario, the TQPT takes place without continuous closing of the spectral gap, which abruptly "inverts" across the transition. Interestingly, this result extends to the 3D case a recent finding of ours for quantum spin Hall insulators in $2 \mathrm{D}^{25}$. Reducing the crystal-field splitting, the strong topological insulator, in the present case, turns into a weak topological insulator through a transition which remains continuous, irrespective of the strength of the interaction. This effect has no analog in the previously studied 2D model.

This sequence of transitions occurs for every value of the Hund's exchange coupling $J$, even if the shape of the phase diagram in the $U-M$ plane is strongly influenced by the presence of the exchange coupling. A qualitative effect of $J$ is that it allows for a topologically trivial Mott insulating state for large values of the interaction strength. This leads to one further TQPT which separates the weak 3D TI from the trivial Mott Insulator (MI). We finally point out that, in a tiny slice bordering the transition line between the 3D TI and MI, we observe the appearance of an anomalous strong "Mottlike topological insulator". Such anomalous strong TI is continuously connected to the MI but separated by a first-order discontinuity from the weak 3D TI state.

The article is organized as follows. In Sec. II, we introduce the non-interacting model for the 3D TI and dis- cuss its solution within the framework of DMFT. We also briefly describe the calculation of the $\mathbb{Z}_{2}$ topological invariant with and without interactions. The multiple phases of the non-interacting model are reviewed in Sec. III. The effects of the electronic correlations on the model solution are discussed in Sec. IV, where we also present the phase diagrams of the full model. In Sec. V, we illustrate the ways the different topological phase transitions occur. Finally, the transition to the Mott insulator is described in Sec. VI which precedes the conclusions of the paper.

\section{MODEL}

We consider a two-orbital Fermi-Hubbard model defined on a three-dimensional cubic lattice. The model Hamiltonian reads:

$$
H=\sum_{\mathbf{k}} \Psi_{\mathbf{k}}^{\dagger} \hat{\mathbf{H}}(\mathbf{k}) \Psi_{\mathbf{k}}+H_{\mathrm{int}}
$$

where the spinor $\Psi_{\mathbf{k}}=\left(\begin{array}{llll}c_{1 \mathbf{k} \uparrow} & c_{2 \mathbf{k} \uparrow} & c_{1 \mathbf{k} \downarrow} & c_{2 \mathbf{k} \downarrow}\end{array}\right)$ collects all the operators $c_{\mathbf{k} l \sigma}\left(c_{\mathbf{k} l \sigma}^{\dagger}\right)$, which annihilate (create) an electron at the orbital $l=1,2$ with momentum $\mathbf{k}$ and spin $\sigma$. In order to write the explicit expression for $\hat{\mathbf{H}}(\mathbf{k})$ let us introduce the $4 \times 4 \Gamma$-matrices, defined as follows:

$$
\begin{aligned}
& \Gamma_{0}=\mathbb{1} \otimes \mathbb{1}=\left[\begin{array}{ll}
\mathbb{1} & \mathbf{0} \\
\mathbf{0} & \mathbb{1}
\end{array}\right] \\
& \Gamma_{1}=\sigma_{z} \otimes \tau_{x}=\left[\begin{array}{cc}
\tau_{x} & \mathbf{0} \\
\mathbf{0} & -\tau_{x}
\end{array}\right] \\
& \Gamma_{2}=-\mathbb{1} \otimes \tau_{y}=\left[\begin{array}{cc}
-\tau_{y} & \mathbf{0} \\
\mathbf{0} & -\tau_{y}
\end{array}\right] \\
& \Gamma_{3}=\sigma_{x} \otimes \tau_{x}=\left[\begin{array}{cc}
\mathbf{0} & \tau_{x} \\
\tau_{x} & \mathbf{0}
\end{array}\right] \\
& \Gamma_{4}=\sigma_{y} \otimes \tau_{x}=\left[\begin{array}{cc}
\mathbf{0} & -i \tau_{x} \\
i \tau_{x} & \mathbf{0}
\end{array}\right] \\
& \Gamma_{5}=\mathbb{1} \otimes \tau_{z}=\left[\begin{array}{cc}
\tau_{z} & \mathbf{0} \\
\mathbf{0} & \tau_{z}
\end{array}\right]
\end{aligned}
$$

where $\tau_{x, y, z}$ and $\sigma_{x, y, z}$ are the Pauli matrices respectively in orbital and in spin space and $\mathbb{1}$ is the unit matrix. In terms of these $\Gamma_{i}$ matrices we have:

$$
\begin{aligned}
& \hat{\mathbf{H}}(\mathbf{k})=M(\mathbf{k}) \Gamma_{5}+\lambda \sin \left(k_{\mathrm{x}}\right) \Gamma_{1}+ \\
& \lambda \sin \left(k_{\mathrm{y}}\right) \Gamma_{2}+\lambda \sin \left(k_{\mathrm{z}}\right) \Gamma_{3}
\end{aligned}
$$

with $M(\mathbf{k})=M-\epsilon\left[\cos \left(k_{x}\right)+\cos \left(k_{y}\right)+\cos \left(k_{z}\right)\right]$. This term of the model Hamiltonian would describe a system of two bands of width $W=6 \epsilon$, hybridizing with an amplitude $\lambda$ and separated by a crystal-field splitting $2 M$. In the following we shall set $\epsilon$ as our energy unit. In addition we fix $\lambda=0.3$ for definiteness having checked that qualitatively similar results can be obtained for different values of $\lambda$. Throughout this paper we consider a total density of two electrons per site, which corresponds to a global 


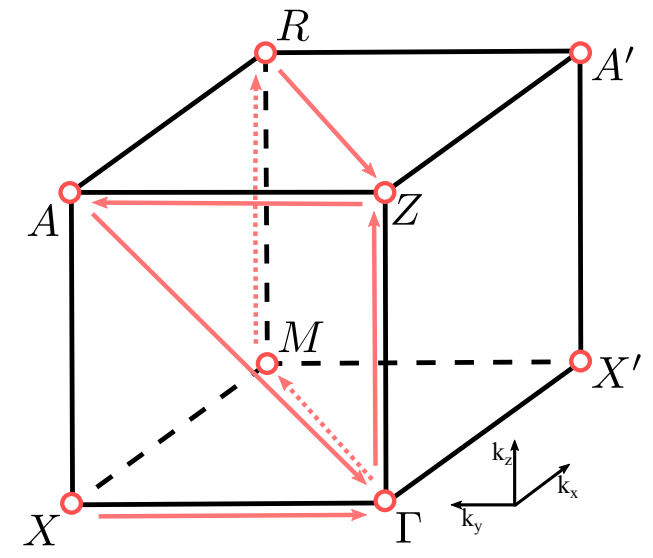

Figure 1. (Color online) Unit cell of the cubic reciprocal lattice. The arrows indicate the three dimensional path in the reciprocal space used to depict the bands in the next Fig.2

half-filling of our band-structure and leads to a particlehole symmetry.

The second term of the model Hamiltonian (1) describes the screened Coulomb repulsion. We assume a local interaction with a full orbital structure, namely inter- and intra-orbital repulsion and the Hund's coupling $J$. This describes the exchange effect favoring high-spin configurations, which correspond to the two electrons occupying different orbitals. More explicitly the interaction is:

$$
\begin{aligned}
H_{\mathrm{int}}= & U \sum_{\mathbf{i} l} n_{\mathbf{i} l \uparrow} n_{\mathbf{i} l \downarrow}+(U-2 J) \sum_{\mathbf{i} l \neq l^{\prime}} n_{\mathbf{i} l \uparrow} n_{\mathbf{i} l^{\prime} \downarrow} \\
& +(U-3 J) \sum_{\mathbf{i} l \neq l^{\prime}}\left(n_{\mathbf{i} l \uparrow} n_{\mathbf{i} l^{\prime} \uparrow}+n_{\mathbf{i} l \downarrow} n_{\mathbf{i} l^{\prime} \downarrow}\right)
\end{aligned}
$$

where $U$ is the strength of the electron-electron interaction and $n_{\mathbf{i} l \sigma}=c_{\mathbf{i} l \sigma}^{\dagger} c_{\mathbf{i} l \sigma}$ is the local density for the orbital $l$ and spin $\sigma$ and $c_{\mathbf{i} l \sigma}=2 \pi / V \sum_{\mathbf{k}} e^{-i \mathbf{k} \cdot \mathbf{i}} c_{\mathbf{k} l \sigma}$. We notice that this Hamiltonian only contains the "densitydensity" part of the Hund's exchange and neglects the so-called pair-hopping and spin-flip terms ${ }^{49}$.

DMFT solution - We investigate the solution of the model (1) using Dynamical Mean Field Theory (DMFT). Within DMFT the quantum many-body lattice Hamiltonian (1) is mapped onto an effective quantum impurity problem. The impurity site features all the local interactions of the original problem. In our case, it corresponds to a two-orbital impurity with interactions of the same form of Eq. (4) coupled to an effective bath. The bath is a frequency dependent quantity whose functional form is obtained self-consistently by imposing that the impurity problem reproduces the local physics of the lattice problem. The DMFT mapping therefore approximates the self-energy of the lattice problem with a momentumindependent one obtained from the solution of the effective quantum impurity problem. In terms of this function the self-consistency condition reads

$$
\begin{aligned}
\hat{\mathcal{G}}_{0}^{-1}(z) & =\sum_{\mathbf{k}}[(z+\mu) \hat{\mathbb{1}}-\hat{\mathbf{H}}(\mathbf{k})-\hat{\boldsymbol{\Sigma}}(z)]^{-1}+\hat{\boldsymbol{\Sigma}}(z) \\
& =\hat{\mathbf{G}}_{\mathrm{loc}}^{-1}(z)+\hat{\boldsymbol{\Sigma}}(z)
\end{aligned}
$$

for $z \in \mathbb{C}$. Equation (5) relates the Weiss Field $\hat{\mathcal{G}}_{0}^{-1}(z)$, describing the properties of the effective bath, to the local physics of the lattice problem expressed by the local Green's function $\hat{\mathbf{G}}_{\mathrm{loc}}(z)$. All the effects of the interactions are included in the self-energy $\hat{\boldsymbol{\Sigma}}(z)$ which is a $4 \times 4$ matrix. Owing to the symmetries of our model, including the particle-hole symmetry at half-filling, the self-energy becomes diagonal in the spin-orbital basis, and it turns out to acquire the following structure in terms of the $\Gamma$ matrices:

$$
\hat{\boldsymbol{\Sigma}}(z)=\sum_{k>0} g_{k} z^{2 k} \Gamma_{5}+\sum_{k \geq 0} u_{k} z^{2 k+1} \Gamma_{0}
$$

with $g_{k}, u_{k} \in \mathbb{R}$. In particular, the real- and imaginarypart of the Matsubara self-energy $\hat{\boldsymbol{\Sigma}}\left(i \omega_{n}\right)$ satisfies the relation:

$$
\hat{\boldsymbol{\Sigma}}\left(i \omega_{n}\right)=\operatorname{Re} \Sigma\left(i \omega_{n}\right) \Gamma_{5}+i \operatorname{Im} \Sigma\left(i \omega_{n}\right) \Gamma_{0}
$$

which means that the full self-energy is parametrised by a single scalar complex function $\Sigma\left(i \omega_{n}\right)$. Eq. (7) implies that the imaginary part of the self-energy is the same for all the spin and orbital components, while the real part is the same for the two spin components and it has the opposite sign for the two orbitals. As a matter of fact the real part of the scalar self-energy is simply added to the bare splitting $M$. Therefore we can define an effective mass term in terms of the low-frequency limit of the selfenergy $^{23,50,51}$

$$
M_{\mathrm{eff}}=M+\operatorname{Re} \Sigma(\omega=0)
$$

In the following we will show that this quantity controls the location of the topological phase transitions in the presence of interactions.

We solve the effective problem by using an exact diagonalization impurity solver ${ }^{5-54}$. In this scheme the effective bath is discretized to a finite number $N_{b}$ of levels coupled to the $N_{o}=2$ impurity orbitals. The resulting Hamiltonian is solved by means of the Lanczos technique which allows to determine the lowest part of the spectrum as well as the dynamical correlation functions at zero and low temperatures. In this work we performed calculations for $N_{b}=8$ and checked the convergence as a function of $N_{b}$ by comparing with $N_{b}=10$ for selected values of the model parameters.

Topological invariants - Three dimensional topological insulators preserving TRS with $\mathcal{T}^{2}=-1$, are classified by four $\mathbb{Z}_{2}$ topological invariants ${ }^{28}$, here denoted by $\vec{\nu}=\left(\nu_{0} ; \nu_{1} \nu_{2} \nu_{3}\right)$. The latter three numbers $\nu_{i}, i=1 \ldots 3$ are so called weak topological invariants that describe stacks of 2D topological insulators in the same symmetry 


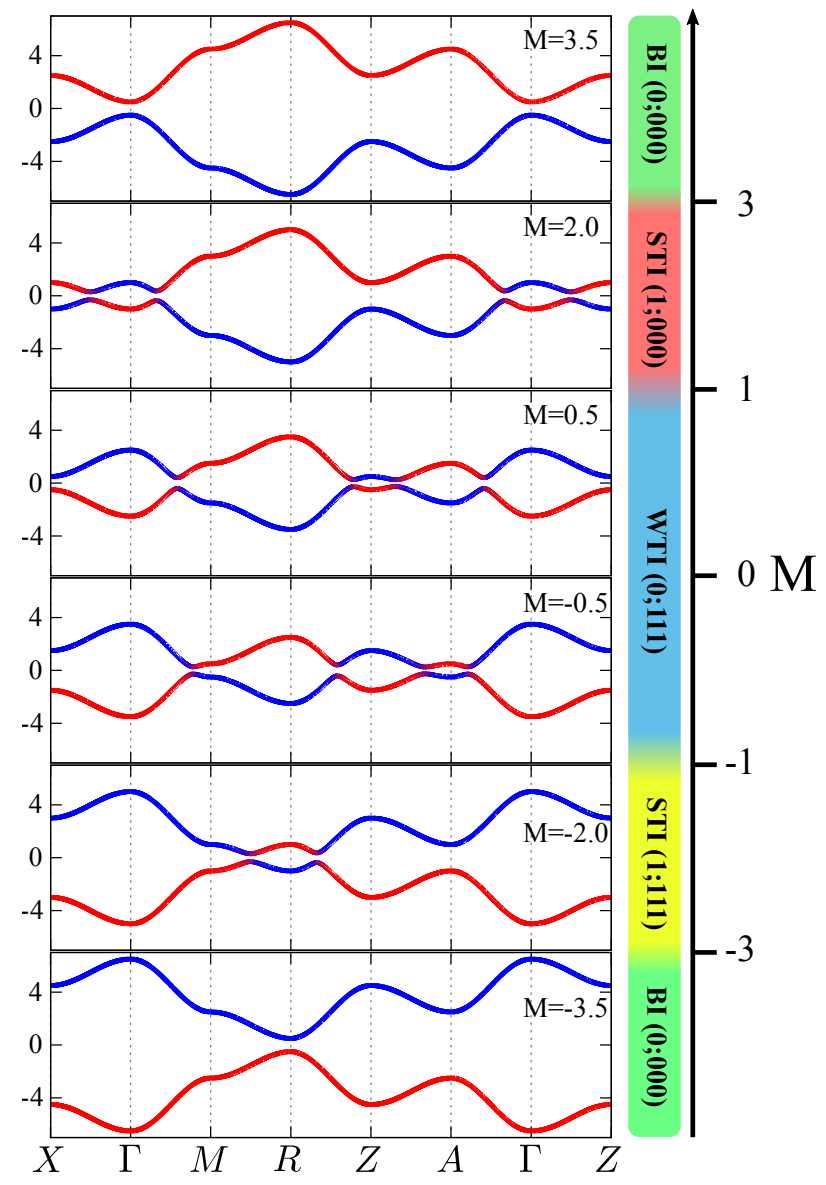

Figure 2. (Color online) Evolution of the non-interacting band-structure (left) as a function of $M$ and consequent "phase diagram" (right) of the model (3). The coloured intervals on the right indicates the different topological phases of the system. The colors of the band structures correspond to the orbital character. The plot also illustrates the difference between the strong topological insulator realized at $M>0$, i.e. $\mathrm{STI}_{\Gamma}$, and the one realized at $M<0$, i.e. $\mathrm{STI}_{R}$.

class. Such weak topological insulators are only defined in lattice systems. By contrast, the strong topological invariant $\nu_{0}$ also exists in continuum systems and exhibits a much stronger robustness against disorder. In the presence of interactions, all these invariants can be generalized to topological properties of the single particle Green's function ${ }^{55-61}$.

As it has been shown in Ref. 62, the practical computation of such interacting invariants formally reduces to that of a non-interacting problem, where the role of the Bloch Hamiltonian is played by the so-called topological Hamiltonian which reads as

$$
\hat{\mathbf{H}}_{\mathrm{t}}(\mathbf{k})=-\hat{\mathbf{G}}^{-1}(\mathbf{k}, \omega=0)=\hat{\mathbf{H}}(\mathbf{k})+\hat{\mathbf{\Sigma}}(\omega=0) .
$$

For the insulating phases considered in this work, the imaginary part of the self-energy is linearly vanishing in $\omega=0$. The only finite contribution thus comes from the real-part $\operatorname{Re} \hat{\boldsymbol{\Sigma}}(\omega \rightarrow 0)$.
If inversion symmetry is not broken, as for our model (1), the definition of the topological invariants is drastically simplified ${ }^{63,64}$. Following Ref. 64, we obtain the eigenstates of $\hat{\mathbf{H}}_{\mathrm{t}}$ at the eight time reversal invariant momenta (TRIM) $K_{i=1 \ldots 8}$ and for the occupied bands $\alpha$. These states can be chosen as eigenstates of the parity operator $\mathcal{P}$ with eigenvalue $p_{\mathrm{i} \alpha}= \pm 1$. The different $\mathbb{Z}_{2}$ invariants $\nu_{c=0, \ldots, 3}$ are then calculated using the relation:

$$
(-1)^{\nu_{c}}=\prod_{\mathrm{i} \in \mathcal{A}_{\mathrm{c}}, \alpha} \sqrt{p_{\mathrm{i} \alpha}}
$$

here the phase convention $\sqrt{-1}=+i$ is used, $c=0, \ldots, 3$ labels the components of the global invariant vector $\vec{\nu}$, and $\mathcal{A}_{c}$ indicates the set of TRIM points as follows (see Fig. 1 for the labels of the TRIM):

$$
\begin{aligned}
& \mathcal{A}_{0}=\left[\Gamma, X^{\prime}, M, X, A, Z, A^{\prime}, R\right] \\
& \mathcal{A}_{1}=\left[X^{\prime}, M, R, A^{\prime}\right] \\
& \mathcal{A}_{2}=[X, A, R, M] \\
& \mathcal{A}_{3}=\left[Z, A^{\prime}, R, A\right],
\end{aligned}
$$

where the location in momentum space of the TRIM points is depicted in Fig.1. Quite intuitively, the sets $\mathcal{A}_{c}, c=0 \ldots 3$ entering the weak topological invariants are confined to $2 \mathrm{D}$ planes with three orthogonal orientations in the 3D Brillouin zone while the strong topological invariant involves all eight TRIM.

\section{TOPOLOGICAL TRANSITIONS IN THE NON-INTERACTING LIMIT}

We begin our analysis from the non-interacting model $U=J=0$. The Hamiltonian (3) describes a threedimensional system undergoing a series of topological quantum phase transitions as a function of the crystalfield splitting term $M$. The band structure along the path in the reciprocal space depicted in Fig.1 for the various phases is reported in Fig.2. For $|M|<1$ the model describes a weak topological insulator (WTI) with a global invariant $\vec{\nu}=(0 ; 111)$.

The smallest direct gap is placed near the point $Z(A)$ for $M>0(M<0)$ in correspondence to a slight inversion of the band character. A more robust band inversion takes place close to the $\Gamma$ point. A Dirac-like gap closure takes place at the points $Z(A)$ for $M=1(M=-1)$.

For values of $M$ in the range $1<|M|<3$ there is a transition to a strong topological insulating (STI) state. In the $M>0$ region such state is characterized by a global invariant $\vec{\nu}=(1 ; 000)$, while for $M<0$ the STI has $\vec{\nu}=(1 ; 111)^{65}$. The values of the topological invariants reflect differences in the band structures: For $M>3$ the smallest direct gap and band inversion takes place around the $\Gamma$ point, while for $M<-3$ these are located in proximity of the $R$ point.

For $|M|=3$ the system undergoes a TQPT from the STI to the trivial band insulator (BI) at $|M|>3$, see 

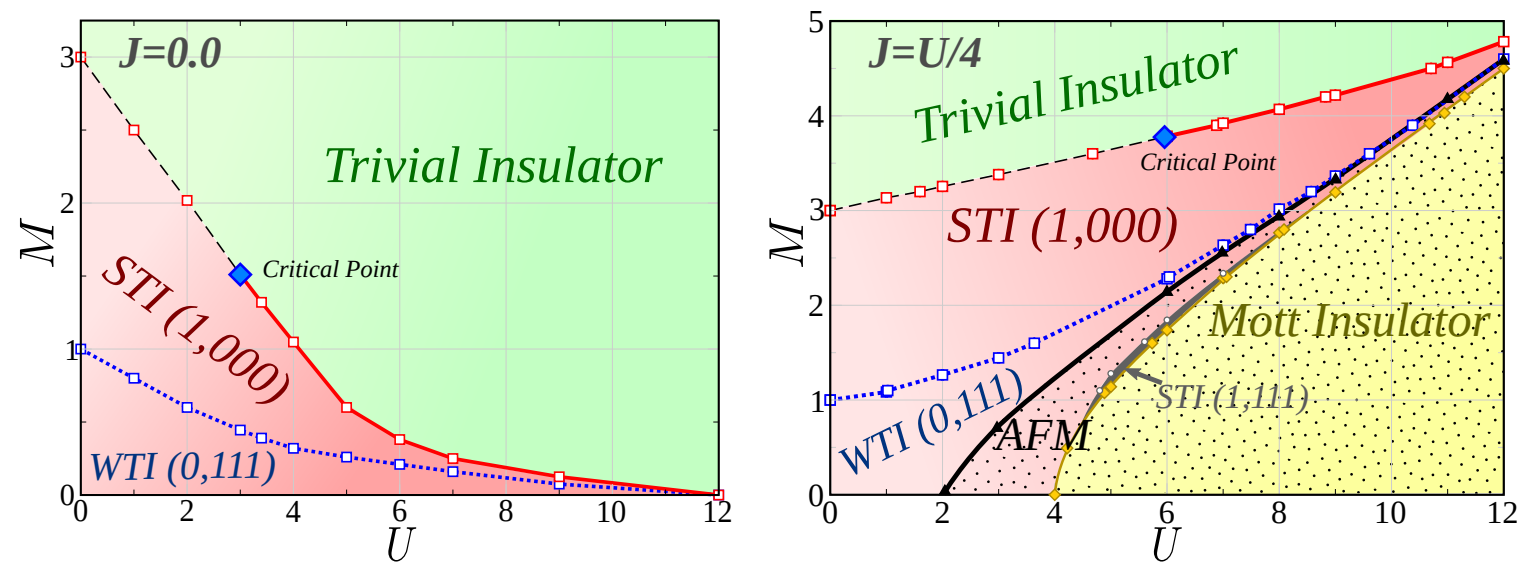

Figure 3. (Color Online) Interacting phase diagram of the three-dimensional model (1) as a function of $M$ and $U$, with $J=0$ (left panel) or $J=U / 4>0$ (right panel). Data are for $\lambda=0.3$. The solid (red) line ends in the Critical Point and is continued beyond this value by the dashed (orange) line. The union of these two lines indicates the boundary between the Trivial Insulator and the Strong Topological Insulator (1,000). The dotted (blue) line indicates the transition from the STI to the weak topological insulator $(0,111)$. In the right panel the solid (yellow) line is the boundary of the Mott Insulating region. The filled (grey) area near the Mott phase shows the re-entrant anomalous STI $(1,111)$ phase, precursor of the Mott transition. The dotted area in the right panel indicates the AFM phase region. The solid (black) line corresponds to the boundary of the AFM phase, which is separated from the non-trivial region by a first-order transition.

Fig.2. At the transition the topological invariant undergoes a sudden change to the trivial value $\vec{\nu}=(0 ; 000)$. The TQPT is continuous with the formation of a Diraclike gap closure at the point $\Gamma(M=3)$ or $R(M=-3)$. For this reason we will indicate with $S T I_{\Gamma}$ and $S T I_{R}$ respectively the strong topological phases for $M>0$ and $M<0$.

\section{INTERACTING PHASE DIAGRAMS}

We now turn our attention to the effects of the interaction on the properties of the TQPT in the model (1). For definiteness we restrict our analysis to the positive values of the splitting $M$. Analogous results can be obtained for the $M<0$ case.

Our results are summarized in the phase-diagrams in the $U-M$ plane reported in Fig.3. The figure compares the phase-diagram in the $U-M$ plane for zero Hund's coupling $J=0$ (left panel) to that for $J=U / 4$ (right panel). In both diagrams one clearly sees that the two transitions of the non-interacting system occurring from $M=3$ (between BI and STI) and $M=1$ (between STI and WTI) are continued into boundary lines that extend in the diagram as $U$ is increased. The phase boundaries have been drawn according to explicit evaluation of the topological invariants from the single-particle Green's function ${ }^{55-59}$.

However, at least two macroscopic differences appear immediately: (i) a Mott insulating phase is obtained only for finite $J$ and (ii) the phase boundaries separating respectively BI from STI and STI from WTI have a completely different behavior as a function of $U$, where the critical $M$ decreases as a function of $U$ in the absence of
$J$, and it has the opposite behavior for finite $J$. We notice that for a wide range of $J$ the phase diagram is similar to that of $J=U / 4$, with the only exception of very small values of $J / U$, where the boundary lines first decrease (as for $J=0$ ) and then increase (as for $J=U / 4$ ) as a function of $U^{66}$.

We can understand these differences analyzing the realpart of the self-energy Re $\hat{\boldsymbol{\Sigma}}$, which renormalizes the bare splitting between the orbitals. In Fig.4 we report the evolution of $\operatorname{Re} \hat{\boldsymbol{\Sigma}}$ as $U$ is increased for $J=0$ (left panel) and $J>0$ (right panel).

The difference is apparent: for $J=0$ the self-energy is positive and increases with $U$, while for $J>0$ it is a negative quantity whose absolute values grows with $U$. This implies that in the absence of $J$ the interaction effectively enhances the crystal-field splitting, while an opposite effect takes place for finite $J$. This is easily understood since the Hund's coupling favours configurations in which the electrons populate the different orbitals to maximize the spin (One can visualize this very simply in the atomic limit where all the kinetic terms are neglected), while in the absence of $J$ intra- and inter-orbital repulsion are identical and the electrons can freely populate the orbitals.

As a result, for $J=0$ the interactions lead to a very large effective splitting, which favours a BI configuration in which only one orbital is populated. For this reason the boundary lines decrease as a function of $U$. On the contrary, for finite $J$ and large interaction a weak effective splitting leads to a Mott insulator with one electron per orbital and increases the threshold to reach the topological transitions.

The presence of large local moments in the high-spin 


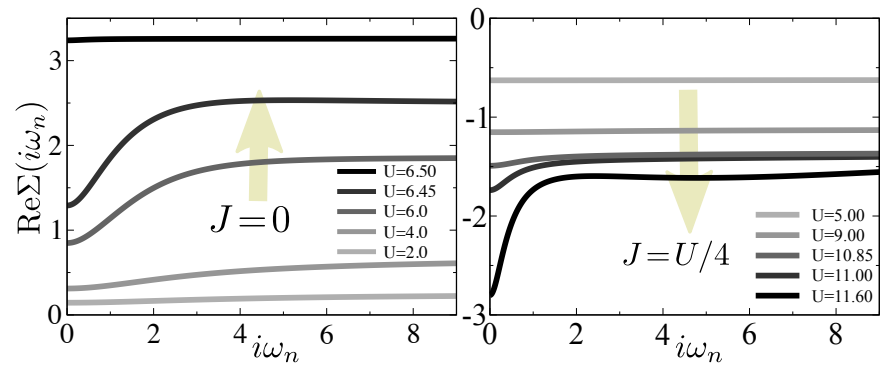

Figure 4. (Color Online) Real part of the scalar Matsubara self-energy $\operatorname{Re} \Sigma\left(i \omega_{n}\right)$ for an increasing interaction strength $U$. Data are shown for $M=0.3$ with $J=0$ (left panel), or $M=4.5$ with $J=U / 4$ (right panel). The figure illustrates the opposite algebraic sign of the self-energy in the two investigated cases. It also points out the pronounced frequency dependence at low-energy.

Mott insulating region favours the onset of magnetic ordering. We verified that, as expected, our model solution is unstable towards the formation of a topologically trivial G-type antiferromagnetic (AFM) state for large $U$. We computed the boundary of the resulting AFM region in the phase diagram (see Fig.3) when allowing for a broken symmetry solution. In addition we found that the formation of the AFM state takes place through a first-order transition, with the discontinuous formation of a finite order parameter. Interestingly the AFM region does not hide the TQPT between BI and $\mathrm{STI}_{\Gamma}$.

In addition to the $J$-dependence, the evolution of the self-energy of Fig.4 reveals another important feature. In both cases for small values of $U$ the real part of the self-energy is essentially constant as a function of the imaginary frequency. This is consistent with a HartreeFock solution (or static mean-field) which can indeed obtained at lowest order in a diagrammatic expansion. As $U$ increases $\operatorname{Re} \hat{\boldsymbol{\Sigma}}$ acquires a more and more pronounced frequency dependence. Roughly speaking the dynamical nature of the interaction effects is measured by the difference between the low-frequency limit, in which the dynamical correlation effects are dominant and the largefrequency limit, that we label as a static mean-field value $\hat{\boldsymbol{\Sigma}}_{\mathrm{MF}}=\operatorname{Re} \hat{\boldsymbol{\Sigma}}\left(i \omega_{n} \rightarrow \infty\right)$. In Fig.5 we show the behavior of the correlation strength $\Xi$, which we define as:

$$
\Xi=\frac{\operatorname{Tr}\left[\Gamma_{5} \operatorname{Re} \hat{\boldsymbol{\Sigma}}(0)-\Gamma_{5} \hat{\boldsymbol{\Sigma}}_{\mathrm{MF}}\right]}{\operatorname{Tr}\left[\Gamma_{5} \hat{\boldsymbol{\Sigma}}_{\mathrm{MF}}\right]},
$$

as a function of $M$ and for different values of $U$ and $J=U / 4$.

For a fixed value of the interaction $U$ the system is driven through a TQPT by decreasing $M$ below a given critical value. In the weakly interacting regime the degree of correlation $\Xi$ remains small and smooth across the TQPT (for smaller values of $U$ than those considered in the figure, $\Xi$ is even more structureless at the topological transition). This behavior changes dramatically in the

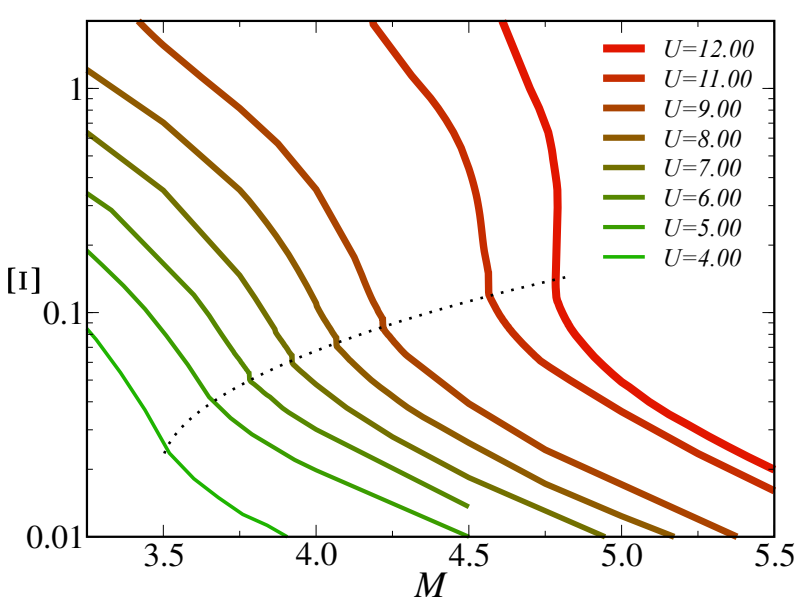

Figure 5. (Color Online) Evolution of the correlation strength measured by $\Xi$ as a function of $M$ and for increasing values of $U$. All data are for $J=U / 4$. The dotted line indicate the transition points.

strongly interacting regime: The curves of $\Xi$ at constant $U$ become divided into to distinct parts: the large- $M$ piece resembles the weak dependence of the small- $U$ region. For $M$ below the TQPT (marked in the figure by a dotted line) $\Xi$ gets instead very rapidly large indicating a strong degree of many-body character of the solutions. This characteristic behaviour of $\Xi$ reflects a simple physical effect: The fully polarized BI with two electrons in the lowest orbital bands is essentially unaffected by the strength of the interaction. On the other hand the topological $\mathrm{STI}_{\Gamma}$ and WTI region do not have a full orbital polarization (hence a higher degree of hybridization). As such they react more vigorously to the presence of interaction, evolving from a weakly perturbed regime to a strongly correlated topological state, as evidenced by $\Xi$.

\section{TOPOLOGICAL QUANTUM PHASE-TRANSITIONS.}

We now study in detail the effect of correlations on the two transitions connecting respectively the BI from the $\mathrm{STI}_{\Gamma}$ and the latter from the WTI.

As we mentioned in Sec. II, the transition lines can be traced according to the evolution of the effective mass term $M_{\text {eff }}$, which is the only way in which the interactions influence the value of the topological invariants.

The TQPT from the trivial $\mathrm{BI}$ to $\mathrm{STI}_{\Gamma}$ is defined by the condition $M_{\text {eff }}=3$ which generalizes the condition $M=3$ valid for the non-interacting case, and naturally reduces to it for $U=0$ when the self-energy vanishes. The whole $M_{\text {eff }}=3$ line is characterized by a change of the bulk topological invariant $\vec{\nu}$ from $(0,000)$ in the trivial $\mathrm{BI}$ phase to $(1,000)$ in the $\mathrm{STI}_{\Gamma}$. However the nature of such transition changes dramatically from the weak to the strong interaction regime, as evidenced by the behavior of $M_{\text {eff }}$ reported in Fig.6 for $J=0$ and 
$J>0$. In both cases, for low values of the interaction $U$ the transition remains continuous, similarly to the noninteracting regime discussed in Sec. III. This reflects the fact that, if the correlation strength is small, the TQPT can be very well described within a Hartree-Fock picture, as already indicated by the behavior of $\Xi$. In this effective single-particle picture a change in the mass term $M$ perfectly compensates the effects of the interaction $U$, ultimately leading to a continuous, non-interacting like, TQPT. This behavior is illustrated in Fig.6 for the smallest values of $U$ for which the crossing of the $M_{\mathrm{eff}}=3$ line is continuous.

By increasing the interaction strength $U$ above the critical value $U>U_{c}$, the degree of correlation of the $\mathrm{STI}_{\Gamma}$ is no longer negligible. Consequently, a renormalized single-particle description of the TQPT breaks down. In particular, the strong dynamical dependence of the selfenergy in this regime does not allow to compensate the effects of the interactions by means of a static change of $M$. The transition is still positioned at $M_{\text {eff }}=3$ but the ground states in the trivial $\mathrm{BI}$ and in the more correlated $\mathrm{STI}_{\Gamma}$ can indeed no longer be continuously connected across the TQPT. As a consequence, a first-order jump is required to move from one phase to the other. The existence of such a first-order TQPT is demonstrated in Fig.6, where we show the evolution of $M_{\text {eff }}$ for values of $U$ above the critical point. As the figure shows, in such regime the effective mass term displays a discontinuity at $M_{\text {eff }}=3$, irrespective of the value of the Hund's coupling $J$. As a further hallmark of the first-order character of the correlated TQPT, we show the hysteretic cycle across the transition in the inset to Fig.6.

This finding shows that electron-electron correlations profoundly change the nature of the topological phase transitions, similarly to what we already found in two dimensions ${ }^{25}$ for quantum spin Hall insulators. In the three-dimensional system, the transition from the BI to the $\mathrm{STI}_{\Gamma}$ is therefore continuous for small values of $U$ but it becomes of first-order upon crossing the critical point, at which the orbital fluctuations become critical ${ }^{25}$. The breakdown of the continuous character of the TQPT in Hamiltonian models similar to our has been recently investigated by means of a renormalization group analysis in Ref. 67.

Our findings resembles the marginal quantum criticality scenario proposed in Refs. $68-70$, where a critical point separates a first-order line from a second-order one. However, a thorough analysis of the scaling properties near the critical point are required to establish a more detailed connection, which is however beyond the scope of this work.

Interestingly, while the increased level of correlation strength dramatically affects the TQPT, it has a very weak impact on the other topological transition, the one from $\mathrm{STI}_{\Gamma}$ to the WTI. Here the change of the bulk invariant from $\vec{\nu}=(1,000)$ to $\vec{\nu}=(0,111)$ is always accompanied by a continuous evolution at $M_{\text {eff }}=1$. To the best of our accuracy we did not find any evidence
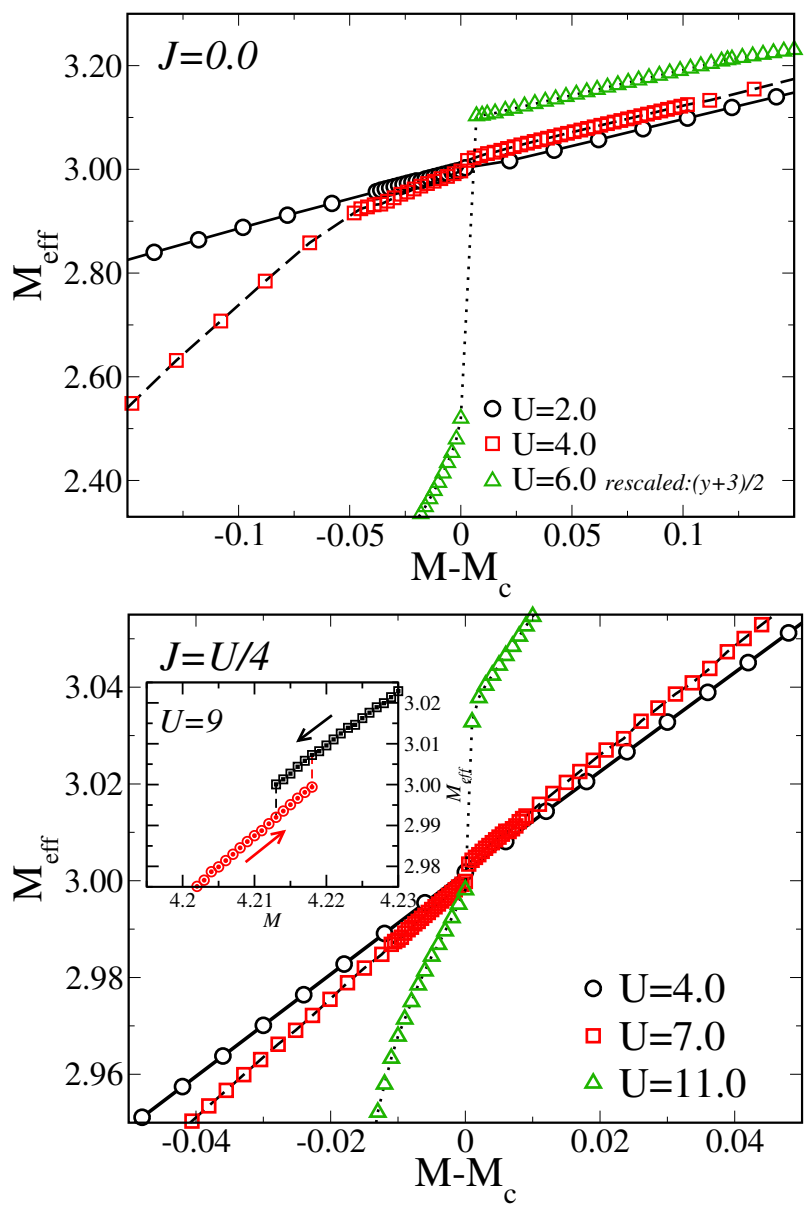

Figure 6. (Color Online) Evolution of the effective Mass term $M_{\text {eff }}$ as a function of the bare mass $M$. Panel (a)-(b) illustrates the transition from Trivial Band Insulator to Strong Topological Insulator for $J=0($ a) and $J>0)($ b). Inset: Hysteresis loop of $M_{\text {eff }}$ for $U=9$ and $J=U / 4$.

of discontinuity, even at the largest investigated value of the interaction strength $U$. This behavior can be understood noting that, although topologically distinct, the two non-trivial states react in a very similar way to the large interaction having a comparable degree of orbital hybridization. Thus, unlike the $\mathrm{BI}$ to $\mathrm{STI}_{\Gamma} \mathrm{TQPT}$, in this case it is always possible to continuously transform the $\mathrm{STI}_{\Gamma}$ ground state into that of the WTI.

\section{A. Absence of gap closing transition.}

As we discussed in the initial sections II and III, in the non-interacting limit the TQPT are always continuous. This ultimately depends on the fact that the band structure of the system evolves smoothly with respect to changes in the model parameters, e.g. $M$, as long as the energy gap is preserved. In presence of large interaction however this scenario is challenged and a different behavior can be envisaged. We shall now show how the change 

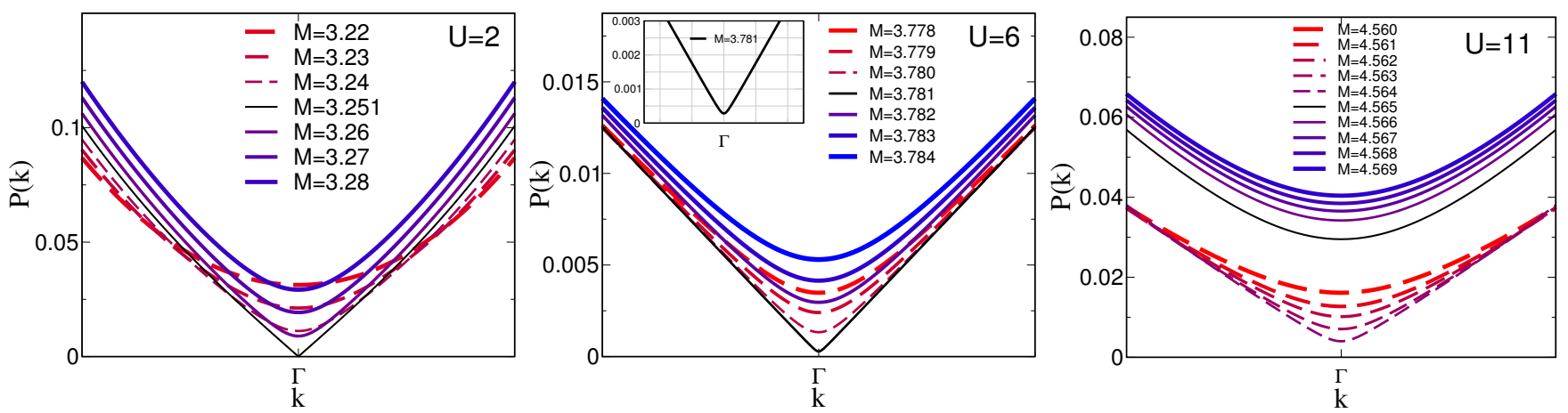

Figure 7. (Color Online) Absence of bulk gap closing: Evolution of the Green's functions poles, i.e. the band dispersions. We find a continuous transition at weak interaction $(U=2)$, contrasted by the absence of bulk gap closure at strong interaction $(U=11)$.

in the TQPT character across the phase-diagram affects the spectral properties of the transitions.

Our results about the absence of a gap closing TQPT are summarized in Fig.7. Each plot reports the behavior of the poles $P(\mathbf{k})$ of the Green's function $\hat{\mathbf{G}}(\mathbf{k}, \omega)=$ $[(\omega+i \eta+\mu) \hat{\mathbf{1}}-\hat{\mathbf{H}}(\mathbf{k})-\hat{\mathbf{\Sigma}}(\omega)]^{-1}$, determined by the con$\operatorname{dition} \operatorname{det}[\hat{\mathbf{G}}(\mathbf{k}, \omega)]=0$, near the point $\Gamma=(0,0,0)$ and along the path $[-R, \Gamma, R]$. The curve $P(\mathbf{k})$ sets the position of the electronic excitations, it determines the spectrum of the system and helps determining the properties of the bulk spectral gap. From left to right, the figure shows the evolution of the poles as a function of $M$ across the BI-STI ${ }_{\Gamma}$ transition for three values of the interaction strength $U$ : Below, at, and above the critical point.

For small values of $U$ the transition occurs through the formation of a Dirac cone at the $\Gamma$ point, as expected by the continuity of the TQPT with respect to the non-interacting case. On the contrary, for $U>U_{c}$ the topological transition takes place without a closure of the spectrum, as we show in the panels (b)-(c). Thus, at the strongly correlated transition point the gap jumps discontinuously from the trivial to the topological value, although in proximity of the critical point it becomes very small. The absence of any gap-less state separating the two phases across the TQPT is a remarkable effect induced by the strong electronic correlation.

\section{MOTT TRANSITION.}

One of the qualitative changes brought in by a finite value of the Hund's coupling is the onset of a Mott insulating region for large $U$, as we reported in Fig.3 (right panel). Indeed, in this case the system favours the formation of an orbital un-polarized state, ultimately leading to an instability towards the high-spin Mott phase as soon as the equal occupation of each orbital is reached ${ }^{25,66}$. Such a high-spin Mott insulator cannot be realized in the $J=0$ case, where the strong interaction favours the formation of an orbitally polarized configuration, i.e. a trivial band insulator (see Fig.3).

In Fig.8(a) we demonstrate the existence of a Mott transition for large values of $U$. The formation of a Mott insulating state is signaled by the divergence of the imaginary part of the self-energy at the chemical potential. Thus we can identify the onset of the Mott phase by means of the reduction to zero of the renormalization constant $Z$, defined as $Z=\left[1-\partial \Sigma(\omega) /\left.\partial \omega\right|_{\omega \rightarrow \mu}\right]^{-1}$. The different phases of the system have an insulating character, thus we can not interpret $Z$ in terms of a quasiparticle weight. As our results show, for a fixed value of the interaction $U$, we can drive the $Z$ towards zero by decreasing the mass term $M$, entering this way the high-spin Mott phase (see Fig.3). The Mott transition has a characteristic first-order behavior, with all the observables showing a discontinuity at the transition point and a small hysteretic behavior (not shown). The presence of such a discontinuity is evident in the behavior of $Z$ for a small enough value of $U$, but it becomes smaller approaching the large $U$ regime. The Mott transition line ends in a triple point, beyond which any correlated topological state disappears, opening the way to a direct transition from the polarized trivial BI to the high-spin Mott state ${ }^{25,66}$.

\section{A. Small region of anomalous $\mathbf{S T I}_{R}$ phase.}

In correspondence to the divergence of the imaginary part of the self-energy at the Mott point, the real part $\operatorname{Re} \hat{\boldsymbol{\Sigma}}\left(i \omega_{n}\right)$ becomes large and strongly frequency dependent (see Fig.8(c)). This behavior is necessary for the opening and the stabilization of the Mott gap. The progressive localization of the electrons obliterates the topological properties of the system, which are related to the low-energy band structure. In other words the large negative (for $J>0$ ) values of $\operatorname{Re} \hat{\boldsymbol{\Sigma}}(0)$ dominate the spectrum of the topological Hamiltonian Eq. (9), giving rise to a trivial topological invariant $\vec{\nu}=(0 ; 000)$ for the Mott phase.

Interestingly, we find that for intermediate values of 

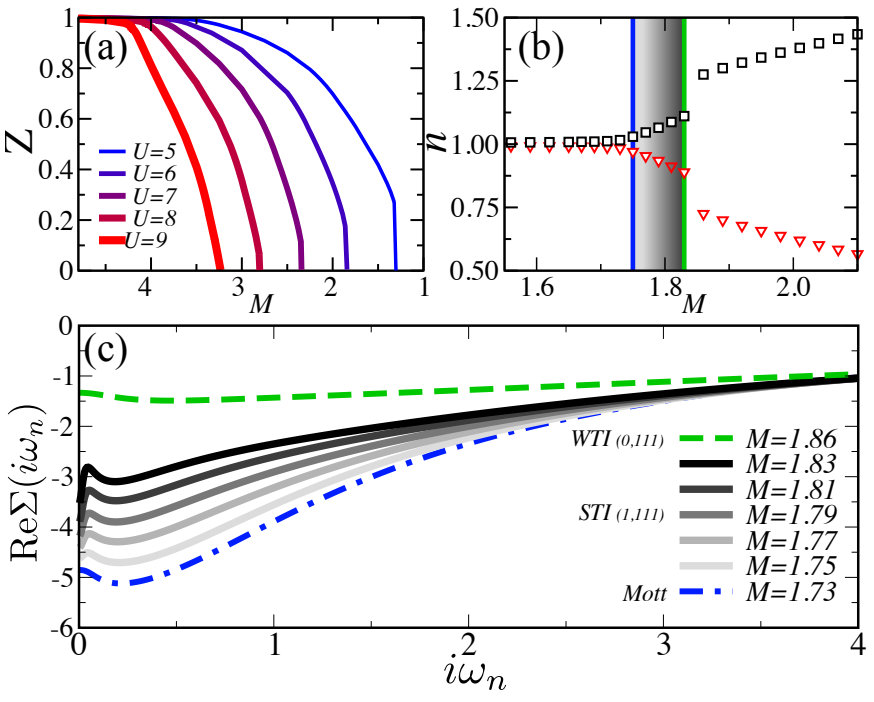

Figure 8. (Color Online) (a) Renormalization constant $Z$ as a function of $M$. Data are for increasing values of $U$ and for $J=U / 4$. Formation of the Mott insulating state is signaled by the vanishing of $Z$. The transition is generically of the first-order. The discontinuity reduces approaching the triple point. (b) Orbital occupation across the WTI-Mott Insulator transition through the $\mathrm{STI}_{R}$. First-order jump takes place at WTI-STI $R$ transition. (c) Real part of the scalar Matsubara self-energy $\operatorname{Re} \Sigma\left(i \omega_{n}\right)$ for increasing values of $M$. Data are for $U=6$ and $J=U / 4$. The plot illustrates how large self-energy lead to the formation of the anomalous $\mathrm{STI}_{R}$ phase.

both the interaction $U$ and mass $M$ the system admits an anomalous topological phase in proximity of the Mott transition point. In particular, our results show that the formation of the Mott insulator can be preceded by a TQPT to a STI state with $\vec{\nu}=(1,111)$, i.e. $\mathrm{STI}_{R}$, which coating the boundary line separates the WTI from the Mott insulating region. The existence of such tiny region near the Mott phase is reported in the phasediagram in Fig.3 (right panel). The origin of such anomalous $\mathrm{STI}_{R}$ state in the $M>0$ can be understood from the behavior of the self-energy in proximity of the Mott point. Near the Mott region $\operatorname{Re} \hat{\boldsymbol{\Sigma}}\left(i \omega_{n}\right)$ can attain values which are large enough to compensate the initial $M$, but such that the effective mass is pushed into the range $M_{\text {eff }} \in[-1,-3]$. For any such value of $M_{\text {eff }}$ the spectrum of the topological Hamiltonian would correspond to a global topological invariant of $\vec{\nu}=(1 ; 111)$, i.e. give rise to a $\mathrm{STI}_{R}$ phase.

Unexpectedly, the anomalous $\mathrm{STI}_{R}$ phase is separated from the WTI by a first-order like transition, while it is continuously connected with the Mott insulating region. This behavior is well visible in the Fig.8(b), where we report the orbital occupations as a function of $M$ in the region near the anomalous $\mathrm{STI}_{R}$ phase. As the plot shows, for small $M$ the occupations are both equal and identical to one, as expected in the Mott phase. By increasing $M$ above the Mott transition value $M>M_{\mathrm{MT}}$ the occupations continuously deviates from one, signaling the onset of the anomalous $\mathrm{STI}_{R}$ phase. This behavior is suddenly arrested by increasing $M$ above a second critical value $M>M_{\mathrm{R}}$, at which a first-order discontinuity in the occupations denotes the TQPT to the WTI phase.

\section{CONCLUSIONS}

We studied the effects of strong electronic correlation on the properties of a paradigmatic model for threedimensional topological insulators. In particular, we considered a local density-density multi-orbital electronic repulsion, in presence of a Hund's coupling $J$ taking into account the tendency of electrons to maximize the total spin orientation while minimizing the orbital polarization. We solved the interacting problem in a nonperturbative way using dynamical mean-field theory. We determined the zero temperature phase-diagrams of the model as a function of the interaction strength $U$ and the crystal-field, or mass term, $M$, both for zero and finite values of the local Hund's' coupling $J$. We explained the specific form of the diagrams in both cases in terms of the different behaviours of the self-energy functions, accounting for the interaction effects at the single-particle level. The existence of a topologically trivial Mott insulating state for $J>0$ and large $U$ is shown.

We point out that, notably, the phase diagrams feature the presence of a critical point on the topological quantum phase-transition line separating the trivial band insulators from the strong topological phase. In addition we unveiled the evolution of the topological transition crossing such critical point. Our findings demonstrate that in the weak-interaction regime the transition remains continuous while in the strong-coupling regime, i.e. beyond the critical point, the transition becomes discontinuous, i.e. a first-order transition.

The main consequence of the first-order character appearing beyond a critical point in the orbital sector is the absence of gap closing. This means that the inversion in the orbital character responsible for the change in the topological invariants is not accompanied by a continuous evolution of the spectral gap, if many-body processes dominate. This is a novel observation that clearly characterizes the transition from the trivial band-insulator to the strong-TI phase. The successive strong-to-weak topological transition is instead always of second-order character, as in the non-interacting band-structure. These results generalize and extend to the three-dimensional case our findings for the topological transition to the quantum spin Hall state in two-dimensions ${ }^{25}$.

In addition, we find the existence of a $(1 ; 111)$ phase, i.e. a strong TI phase which appears before the eventual transition from the weak- to the trivial Mott insulator. An analysis of the orbital occupations in this narrow region of the phase diagram reveals that this phase is separated from the WTI $(0 ; 111)$ by a first-order transition and from the Mott insulating state by a continuous one. Although this phase has the same topological in- 
dices $(1 ; 111)$ as the single-particle $\mathrm{STI}_{R}$ phase at $U=0$ (i.e. $M \in[-1,-3])$, the two can not be directly connected in our phase-diagram without crossing a topological transition line. Indeed, the re-entrant $(1 ; 111)$ phase emerges here as the result of the strong many-body correlation encoded in the dynamical self-energies.

\section{A. Acknowledgments}

G.S. is grateful to Piet Brouwer for useful discussions. M.C. and A.A. acknowledge financial support from the European Research Council under FPO7 Starting Independent Research Grant n.240524 "SUPER BAD". B.T. and G.S. acknowledge financial support by the DFG (SPP 1666 and SFB 1170). J.C.B. acknowledges financial support from the ERC synergy grant UQUAM.
1 A. P. Schnyder, Ryu S., A. Furusaki, and A. W. W. Ludwig, "Classification of topological insulators and superconductors in three spatial dimensions." Phys. Rev. B 78, 195125 (2008).

2 Alexei Kitaev, "Periodic table for topological insulators and superconductors," AIP Conference Proceedings 1134, 22-30 (2009).

3 A. Altland and M. R. Zirnbauer, "Nonstandard symmetry classes in mesoscopic normal-superconducting hybrid structures." Phys. Rev. B 55, 1142 (1997).

${ }^{4}$ C. L. Kane and E. J. Mele, " $Z_{2}$ Topological Order and the Quantum Spin Hall Effect," Phys. Rev. Lett. 95, 146802 (2005).

5 C. L. Kane and E. J. Mele, "Quantum Spin Hall Effect in Graphene," Phys. Rev. Lett. 95, 226801 (2005).

${ }^{6}$ B. Andrei Bernevig, Taylor L. Hughes, and Shou-Cheng Zhang, "Quantum Spin Hall Effect and Topological Phase Transition in HgTe Quantum Wells," Science 314, 17571761 (2006).

7 Markus Knig, Steffen Wiedmann, Christoph Brne, and et al., "Quantum Spin Hall Insulator State in HgTe Quantum Wells," Science 318, 766-770 (2007).

8 M Hohenadler and F F Assaad, "Correlation effects in twodimensional topological insulators," Journal of Physics: Condensed Matter 25, 143201 (2013).

9 Thomas C. Lang, Andrew M. Essin, Victor Gurarie, and Stefan Wessel, " $Z_{2}$ topological invariants in two dimensions from quantum Monte Carlo," Phys. Rev. B 87, 205101 (2013).

10 Hsiang-Hsuan Hung, Lei Wang, Zheng-Cheng Gu, and Gregory A. Fiete, "Topological phase transition in a generalized Kane-Mele-Hubbard model: A combined quantum Monte Carlo and Green's function study," Phys. Rev. B 87, 121113 (2013).

11 Hsiang-Hsuan Hung, Victor Chua, Lei Wang, and Gregory A. Fiete, "Interaction effects on topological phase transitions via numerically exact quantum Monte Carlo calculations," Phys. Rev. B 89, 235104 (2014).

12 Jan Carl Budich, Ronny Thomale, Gang Li, Manuel Laubach, and Shou-Cheng Zhang, "Fluctuation-induced topological quantum phase transitions in quantum spinHall and anomalous-Hall insulators," Phys. Rev. B 86, 201407 (2012).

13 F. Grandi, F. Manghi, O. Corradini, and C. M. Bertoni, "Topological properties of the bond-modulated honeycomb lattice," Phys. Rev. B 91, 115112 (2015).

14 F Grandi, F Manghi, O Corradini, C M Bertoni, and A Bonini, "Topological invariants in interacting quantum spin Hall: a cluster perturbation theory approach," New
Journal of Physics 17, 023004 (2015).

15 Jingxiang Wu, Jean Paul Latyr Faye, David Snchal, and Joseph Maciejko, "Quantum cluster approach to the spinful Haldane-Hubbard model," Phys. Rev. B 93, 075131 (2016).

16 Manuel Laubach, Johannes Reuther, Ronny Thomale, and Stephan Rachel, "Rashba spin-orbit coupling in the KaneMele-Hubbard model," Phys. Rev. B 90, 165136 (2014).

17 S. Rachel and K. Le Hur, "Topological insulators and Mott physics from the Hubbard interaction." Phys. Rev. B 82, 075106 (2010).

18 Yao-Hua Chen, Hsiang-Hsuan Hung, Guoxiong Su, Gregory A. Fiete, and C. S. Ting, "Cellular dynamical meanfield theory study of an interacting topological honeycomb lattice model at finite temperature," Phys. Rev. B 91, 045122 (2015).

19 Qi Chen, Hsiang-Hsuan Hung, Xiang Hu, and Gregory A. Fiete, "Correlation effects in pyrochlore iridate thin films grown along the [111] direction," Phys. Rev. B 92, 085145 (2015).

20 Wei Wu, Stephan Rachel, Wu-Ming Liu, and Karyn Le Hur, "Quantum spin Hall insulators with interactions and lattice anisotropy," Phys. Rev. B 85, 205102 (2012).

21 Tsuneya Yoshida, Satoshi Fujimoto, and Norio Kawakami, "Correlation effects on a topological insulator at finite temperatures," Phys. Rev. B 85, 125113 (2012).

22 Tsuneya Yoshida, Robert Peters, Satoshi Fujimoto, and Norio Kawakami, "Topological antiferromagnetic phase in a correlated Bernevig-Hughes-Zhang model," Phys. Rev. B 87, 085134 (2013).

23 Jan Carl Budich, Bjrn Trauzettel, and Giorgio Sangiovanni, "Fluctuation-driven topological Hund insulators," Phys. Rev. B 87, 235104 (2013).

24 R. Nourafkan, G. Kotliar, and A.-M. S. Tremblay, "Orbital magnetization of correlated electrons with arbitrary band topology," Phys. Rev. B 90, 125132 (2014).

25 A. Amaricci, J. C. Budich, M. Capone, B. Trauzettel, and G. Sangiovanni, "First-Order Character and Observable Signatures of Topological Quantum Phase Transitions," Phys. Rev. Lett. 114, 185701 (2015).

26 S. Raghu, Xiao-Liang Qi, C. Honerkamp, and Shou-Cheng Zhang, "Topological Mott Insulators," Phys. Rev. Lett. 100, 156401 (2008).

27 Andreas Regg and Gregory A. Fiete, "Topological Order and Semions in a Strongly Correlated Quantum Spin Hall Insulator," Phys. Rev. Lett. 108, 046401 (2012).

28 Liang Fu, C. L. Kane, and E. J. Mele, "Topological Insulators in Three Dimensions," Phys. Rev. Lett. 98, 106803 (2007). 
29 J. E. Moore and L. Balents, "Topological invariants of time-reversal-invariant band structures," Phys. Rev. B 75, 121306 (2007).

30 Rahul Roy, "Topological phases and the quantum spin Hall effect in three dimensions," Phys. Rev. B 79, 195322 (2009).

31 Moore Joel E., "The birth of topological insulators," Nature 464, 194-198 (2010), 10.1038/nature08916.

32 Hsieh D., Qian D., Wray L., Xia Y., Hor Y. S., Cava R. J., and Hasan M. Z., "A topological Dirac insulator in a quantum spin Hall phase," Nature 452, 970-974 (2008), 10.1038/nature06843.

33 D. Hsieh, Y. Xia, L. Wray, D. Qian, A. Pal, J. H. Dil, J. Osterwalder, F. Meier, G. Bihlmayer, C. L. Kane, Y. S. Hor, R. J. Cava, and M. Z. Hasan, "Observation of Unconventional Quantum Spin Textures in Topological Insulators," Science 323, 919-922 (2009).

${ }^{34}$ Haijun Zhang, Chao-Xing Liu, Xiao-Liang Qi, Xi Dai, Zhong Fang, and Shou-Cheng Zhang, "Topological insulators in Bi2Se3, Bi2Te3 and Sb2Te3 with a single Dirac cone on the surface," Nat Phys 5, 438-442 (2009).

35 Y. L. Chen, J. G. Analytis, J.-H. Chu, Z. K. Liu, S.-K. Mo, X. L. Qi, H. J. Zhang, D. H. Lu, X. Dai, Z. Fang, S. C. Zhang, I. R. Fisher, Z. Hussain, and Z.-X. Shen, "Experimental Realization of a Three-Dimensional Topological Insulator, Bi2Te3," Science 325, 178-181 (2009).

${ }^{36}$ L. A. Wray et al., " A topological insulator surface under strong Coulomb magnetic and disorder perturbations." Nature Phys. 7, 32-37 (2011).

37 Pesin Dmytro and Balents Leon, "Mott physics and band topology in materials with strong spin-orbit interaction," Nat. Phys. 6, 376-381 (2010).

38 Joseph Maciejko, Victor Chua, and Gregory A. Fiete, "Topological Order in a Correlated Three-Dimensional Topological Insulator," Phys. Rev. Lett. 112, 016404 (2014).

39 Maciejko Joseph and Fiete Gregory A., "Fractionalized topological insulators," Nat Phys 11, 385-388 (2015).

40 Maxim Dzero, Kai Sun, Victor Galitski, and Piers Coleman, "Topological Kondo Insulators," Phys. Rev. Lett. 104, 106408 (2010).

${ }^{41}$ M. Dzero, K. Sun, P. Coleman, and V. Galitski, "Theory of topological Kondo insulators," Physical Review B 85, 045130 (2012).

42 Xiaohang Zhang, N. P. Butch, P. Syers, and et al., "Hybridization, Inter-Ion Correlation, and Surface States in the Kondo Insulator $\mathrm{SmB}_{6}$," Phys. Rev. X 3, 011011 (2013).

43 Feng Lu, JianZhou Zhao, Hongming Weng, Zhong Fang, and Xi Dai, "Correlated Topological Insulators with Mixed Valence," Phys. Rev. Lett. 110, 096401 (2013).

${ }^{44}$ Xiaoyu Deng, Kristjan Haule, and Gabriel Kotliar, "Plutonium Hexaboride is a Correlated Topological Insulator," Phys. Rev. Lett. 111, 176404 (2013).

45 Yi Zhang, Ying Ran, and Ashvin Vishwanath, "Topological insulators in three dimensions from spontaneous symmetry breaking," Phys. Rev. B 79, 245331 (2009).

46 I. F. Herbut and L. Janssen, "Topological Mott Insulator in Three-Dimensional Systems with Quadratic Band Touching." Phys. Rev. Lett. 113, 106401 (2014).

47 Gang Li, Werner Hanke, Giorgio Sangiovanni, and Bjrn Trauzettel, "Interacting weak topological insulators and their transition to Dirac semimetal phases," Phys. Rev. B 92, 235149 (2015).
48 M. Kargarian and G. A. Fiete, "Topological Crystalline Insulators in Transition Metal Oxides." Phys. Rev. Lett. 110, 156403 (2013).

49 In Ref. 23 the robustness of the topological transitions in the Bernevig-Hughes-Zhang-Hubbard model against the pair-hopping and spin-flip terms has been verified.

50 A. I. Poteryaev et al., "Enhanced crystal-field splitting and orbital-selective coherence induced by strong correlations in $\mathrm{V}_{2} \mathrm{O}_{3}$," Phys. Rev. B 76, 085127 (2007).

51 N. Parragh et al., "Effective crystal field and Fermi surface topology: A comparison of $d$ - and $d p$-orbital models." Phys. Rev. B 88, 195116 (2013).

52 Antoine Georges, Gabriel Kotliar, Werner Krauth, and Marcelo J. Rozenberg, "Dynamical mean-field theory of strongly correlated fermion systems and the limit of infinite dimensions," Rev. Mod. Phys. 68, 13-125 (1996).

53 Massimo Capone, Luca de' Medici, and Antoine Georges, "Solving the dynamical mean-field theory at very low temperatures using the Lanczos exact diagonalization," Phys. Rev. B 76, 245116 (2007).

${ }^{54}$ C. Weber, A. Amaricci, M. Capone, and P. B. Littlewood, "Augmented hybrid exact-diagonalization solver for dynamical mean field theory," Phys. Rev. B 86, 115136 (2012).

55 A. N. Redlich, "Parity violation and gauge noninvariance of the effective gauge field action in three dimensions," Phys. Rev. D 29, 2366-2374 (1984).

56 Hiroto So, "Induced Topological Invariants by Lattice Fermions in Odd Dimensions," Progress of Theoretical Physics 74, 585-593 (1985).

57 Kenzo Ishikawa and Toyoki Matsuyama, "A microscopic theory of the quantum Hall effect," Nuclear Physics B 280, 523-548 (1987).

58 G.E. Volovik, "Analog of quantum Hall effect in superfluid 3He film," JETP 67(9), 1804-1811 (1988).

59 V. Gurarie, "Single-particle Green's functions and interacting topological insulators," Phys. Rev. B 83, 085426 (2011).

60 Y.-Y. He, H.-Q. Wu, Z. Y. Meng, and Z.-Y. Lu, "Topological invariants for interacting topological insulators: I. Efficient numerical evaluation scheme and implementations," ArXiv e-prints (2015), arXiv:1510.07816 [cond-mat.str-el].

61 Y.-Y. He, H.-Q. Wu, Z. Y. Meng, and Z.-Y. Lu, "Topological invariants for interacting topological insulators: II. Breakdown of the Green's function formalism," ArXiv eprints (2015), arXiv:1512.02080 [cond-mat.str-el].

62 Zhong Wang and Shou-Cheng Zhang, "Simplified Topological Invariants for Interacting Insulators," Phys. Rev. X 2, 031008 (2012).

${ }^{63}$ Liang Fu and C. L. Kane, "Topological insulators with inversion symmetry," Phys. Rev. B 76, 045302 (2007).

64 Zhong Wang, Xiao-Liang Qi, and Shou-Cheng Zhang, "Topological invariants for interacting topological insulators with inversion symmetry," Phys. Rev. B 85, 165126 (2012).

${ }^{65}$ Bjrn Sbierski, Martin Schneider, and Piet W. Brouwer, "Weak side of strong topological insulators," Phys. Rev. B 93, 161105 (2016).

66 Philipp Werner and Andrew J. Millis, "High-Spin to LowSpin and Orbital Polarization Transitions in Multiorbital Mott Systems," Phys. Rev. Lett. 99, 126405 (2007).

67 B. Roy, P. Goswami, and J. D. Sau, "Is the transition between topological and trivial insulators always continuous?" ArXiv e-prints (2015), arXiv:1507.00722 [cond- 
mat.mes-hall].

68 Masatoshi Imada, "Universality classes of metal-insulator transitions in strongly correlated electron systems and mechanism of high-temperature superconductivity," Phys. Rev. B 72, 075113 (2005).
69 Takahiro Misawa and Masatoshi Imada, "Quantum criticality around metal-insulator transitions of strongly correlated electron systems," Phys. Rev. B 75, 115121 (2007).

70 Moyuru Kurita, Youhei Yamaji, and Masatoshi Imada, "Phase diagram structure of topological Mott transition for zero-gap semiconductors beyond conventional LandauGinzburg-Wilson scenario," Phys. Rev. B 88, 115143 (2013). 